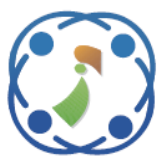

\title{
Improved Precoding Algorithm Design for Downlink Large Scale MU-MIMO System
}

\author{
Jagtar Singh $^{1 *} \quad$ Deepak Kedia $^{1}$ \\ ${ }^{I}$ Department of Electronics and Communication Engineering, \\ Guru Jambheshwar University of Science \& Technology, Hisar, India \\ * Corresponding author's Email: jagtar_nit@rediffmail.com
}

\begin{abstract}
Large Scale Multiuser multiple input multiple output (LS-MU-MIMO) communication system is considered as an encouraging solution to attain greater spectral efficiency for the next-generation wireless communication system. In this paper, we propose an improved design of a precoding algorithm for LS-MU-MIMO communication system. The proposed precoding algorithm is based on Block diagonalization (BD) precoding which is a combination of QR decomposition and Zero forcing channel inversion (ZF-CI) operation named as QR-ZF-BD precoding algorithm. To enhance the performance of LS-MU-MIMO system, the proposed precoding algorithm utilizes ZF method and QR decomposition operation instead of more complex Singular value decomposition (SVD) operation as compared to the conventional Block diagonalization (BD) precoding technique. The proposed precoding algorithm is designed in two stages. In the first stage, the proposed QR-ZF-BD precoding algorithm first utilizes QR decomposition to diminish multiuser interference (MUI), the second stage utilizes ZF based channel inversion (ZF$\mathrm{CI})$ and QR decomposition operation again to further enhance the spectral efficiency of a single cell downlink LSMU-MIMO system. The enhanced spectral efficiencies of values $334 \mathrm{bit} / \mathrm{s} / \mathrm{Hz}$ and $388 \mathrm{bit} / \mathrm{s} / \mathrm{Hz}$ are obtained with our proposed precoding algorithm design when LS-MU-MIMO system having 100 and 200 base station antennas respectively. The numerical results indicate that the proposed QR-ZF-BD precoding algorithm achieves the best spectral efficiency as compared to current LTE-A standard and other recently used conventional linear precoding algorithms.
\end{abstract}

Keywords: Large scale MU-MIMO, Precoding algorithm, Spectral efficiency, QR decomposition, SVD, Block diagonalization $(\mathrm{BD})$.

\section{Introduction}

The increasing demand for wireless services and applications requires radical improvement in future generation wireless communication systems [1]. In past years, the wireless data traffic has been growing fast and is expected to become 200 to 1,000 times up to 2020 [2]. Also report in [3] indicates that demand for higher data rates grows nearly $60 \%$ every year. In future also, the demand for higher data rates will be even more [4]. The exponential growth in data traffic and increase in user terminals require additional throughput and concern of energy consumption by base stations and user terminals. Therefore, the expectations from future generation wireless networks are to meet the demands of high throughput with lesser energy consumption at base stations [1, 5]. To improve spectral efficiency and hence throughput, a well-known method is termed as a MIMO communication technique. Further enhancement in multiplexing gain is achieved by shifting the MIMO system to multiuser MIMO (MU-MIMO) system, where user terminals are served at the same time by the transmitter [6]. Currently, evolving wireless communication networks e.g LTE-Advanced have LS-MU-MIMO technology (also known as Massive MIMO Technology) to further improve the network capacity $[1,7]$. The LS-MU-MIMO technology has been incorporated into 5G new radio standard [8]. Large Scale MU-MIMO is the novel technology to 
enhance the spectral efficiency for the nextgeneration communication systems and drew enormous research attention in previous years $[1,5$, $7,9]$. The higher spectral efficiency is achieved by the beamforming and spatial multiplexing techniques $[1,5,7]$.

The concept of LS-MU-MIMO was proposed by Merzetta in his paper [10]. In LS-MU-MIMO system, the base station is installed with a huge number of antennas, which are used effectively to serve users together in the same frequency-time and employ precoding or detection schemes at the base station. A basic concept of LS-MU-MIMO system is to gain all the advantages of a classical MIMO system but on a large scale. LS-MU-MIMO is the key technology to develop future broadband networks which will be secure, robust, energy efficient and will utilize spectrum efficiently [1]. The canonical form of LS-MU-MIMO system operates on time-division duplexing (TDD) mode and obtain channel state information (CSI) by utilizing pilots transmitted by the user equipments and channel reciprocity [7]. The CSI is required at the transmitter for precoding and receive combining operations. Random channel vectors for different user equipments become nearly orthogonal as compared to conventional MU-MIMO system, which helps favorable propagation in case of LSMU-MIMO system [11].

\subsection{Related work}

Some of the research challenges like channel modelling and measurement [5, 12], design of antenna array [13], channel capacity [7, 11], spectral and energy efficiency $[11,14,15]$, precoding techniques [11, 16] channel estimation [17], pilot contamination [16] have been investigated by the researchers for LS-MU-MIMO communication system.

Our main area of interest in this work is to analyze the impact of precoding techniques on spectral efficiency for LS-MU-MIMO system. Higher spectral efficiency can be attained for LSMU-MIMO system when the base station serves users in the same frequency-time $[9,16]$. Multiuser interference (MUI) becomes a problem in downlink transmission of the signal, which results in reduced performance of an LS-MU-MIMO system. To maximize the performance of LS-MU-MIMO system, MUI should be dealt in an efficient manner. The base station employs precoding to pre-subtract MUI before sending a signal through the channel. The precoding operation at the base station mitigates the effect of inter-user interference and noise which results in significant improvement in spectral efficiency, communication link reliability, quality of service (QoS) and energy efficiency [18, 19].

In LS-MU-MIMO system, most of the previous research works focused on the analysis of linear precoding schemes as in Ref. [7, 11, 15, 23] and of great importance to improve system performance in case of practical applications. In Ref. [14], the performance of downlink LS-MU-MIMO was investigated with $\mathrm{ZF}$ precoding scheme and it was shown that there is an enhancement in BER, spectral efficiency, and outage probability. Authors in Ref. $[7,11]$ analyzed the performance of LS-MU-MIMO system with minimum mean square error (MMSE), Maximum ratio transmission (MRT), Zero-forcing (ZF) and Maximum ratio combining (MRC) precoding schemes. Authors in Ref. [20] presented a performance analysis of large scale MIMO in terms of spectral efficiency where base station employs MRT precoding. In Ref. [21], the authors compared the performance of vector and matrix normalization for MRT and ZF precoding schemes with perfect CSI for cell boundary users. Authors in Ref. [15] studied the spectral efficiency for single cell Massive MIMO systems with MRT precoding. Pilot contamination problem occurs due to intercell interference [16] and needs to be tackled efficiently and pose challenges in the area of precoding and detection algorithms. In Ref. [22, 23], the expression for energy efficiency was derived by considering the energy consumption model and shown that system performance increases with an increase in base station antennas. In Ref. [11], uplink achievable rates and power efficiency was studied with $\mathrm{ZF}$, MRC, and MMSE precoding schemes. In Ref. [24], authors derived expressions for uplink rates were derived by considering perfect and imperfect CSI. Downlink transmission rates of massive MIMO were investigated in Ref. [21] with MRT and ZF precoding schemes and closed-form expressions were derived for low and high SNR regimes. The block diagonalization (BD) precoding scheme eliminates MUI with the improved performance as in Ref. [25, 26] and beneficial when users also having multiple antennas. BD precoding requires higher computation complexity since it depends on the calculation of two SVD operations that are used to compute the precoding matrices $[27,28]$. The complexity of BD precoding is reduced by replacing the first SVD with the QR decomposition but shows the same performance as $\mathrm{BD}$ precoding [29, 30]. There is also some works to reduce the complexity of BD precoding [28-33]. In Ref. [34], the authors investigated a low complexity $\mathrm{BD}$ precoding to eliminate MUI. Authors in Ref. [35] studied BD 
precoding algorithm for multicell MIMO to obtain maximum sum rate. In order to reduce multiuser interference, the outer pre-processor based BD precoding scheme was designed for Massive MIMO system [36]. In Ref. [31], the authors proposed the design of iterative precoder and user scheduling for BD precoder.

Most of the research works discussed above consider linear precoding schemes like MRC, MRT, $\mathrm{ZF}$, and MMSE to enhance spectral efficiency. The prior work on $\mathrm{BD}$ precoding shows the ways to reduce its complexity on its variants but no or little work is observed on the performance improvement in terms of spectral efficiency for LS-MU-MIMO system. In this work, we have proposed QR-ZF based BD precoding algorithm for LS-MU-MIMO communication system with the best performance and low computational complexity as compared to QR-BD and BD precoding algorithms. To best of the authors knowledge, no such prior work has been considered QR-ZF based BD precoding algorithm to enhance spectral efficiency for LS-MU-MIMO downlink system.

\subsection{Summary of contributions}

The aim of our work is to design of improved precoding algorithm and analyze the performance of LS-MU-MIMO system with different precoding algorithms under Rayleigh fading channel when users having two antennas. Our contributions are as follows:

1. We consider a LS-MU-MIMO system where antennas $\mathbf{M}_{t}$ grow large at the base station, with fixed users $K$ and investigate the impact of MRT, ZF, MMSE, QR-BD, BD, and QR-ZF-BD precoding algorithm on spectral efficiency. We consider a Raleigh fading channel in this work. The proposed QR-ZF-BD precoding algorithm is analyzed and compared with the above precoding algorithms.

2. Our results imply that the spectral efficiency increases with the increase in the number of base station (BS) antennas and proposed QR-ZF-BD precoding algorithm show enhanced performance as compared to conventional MRT, ZF, MMSE, BD, QR-BD precoding algorithms.

3. Our results indicate that the spectral efficiency increases with increase in SNR values for all precoding algorithm. The spectral efficiency of $\mathrm{BD}, \mathrm{QR}-\mathrm{BD}$ precoding algorithm is marginally better or equal to that of MMSE, ZF precoding algorithms in all the SNR range. Conversely, MRT precoding show improved performance as compared
Table 1. List of Notations

\begin{tabular}{|c|c|}
\hline$(.)^{\mathrm{H}}$ & $\begin{array}{c}\text { Conjugate and Transpose } \\
\text { (Hermitian) Operator }\end{array}$ \\
\hline $\mathrm{H}$ & Channel matrix \\
\hline $\mathrm{h}$ & Channel vector \\
\hline $\mathrm{H}^{\dagger}$ & Pseudo Inverse of matrix \\
\hline$C N(0,1)$ & $\begin{array}{c}\text { Complex Gaussian with Zero mean } \\
\text { and Variance equal to } 1\end{array}$ \\
\hline $\mathrm{C}^{\mathrm{N}}$ & Complex vectors of N dimensions. \\
\hline $\mathrm{I}$ & Identity Matrix \\
\hline 0 & Zero matrix \\
\hline $\operatorname{diag}\left(\mathrm{H}_{1}\right.$, & Block diagonal matrix \\
\hline $\left.\mathrm{H}_{2} \ldots \ldots . \mathrm{H}_{\mathrm{k}}\right)$ & Inverse of a matrix \\
\hline $\mathrm{H}^{-1}$ & Transpose of a matrix \\
\hline $\mathrm{H}^{\mathrm{T}}$ & Optimal power loading matrix \\
\hline$\wedge$ & Singular value matrix \\
\hline $\boldsymbol{\Sigma}$ & \\
\hline
\end{tabular}

to $\mathrm{ZF}$ precoding at low SNR regime. Simulation results indicate that the QR-ZF-BD precoding algorithm performs better than other precoding algorithms in the entire SNR regime.

The rest of this work is arranged as follows. Section 2 describes the channel model of an LSMU-MIMO over Raleigh fading channel. The proposed QR-ZF-BD algorithm is provided in section 3. Section 4 provides the performance analysis of LS-MU-MIMO system. Results and discussions are presented in section 5. Finally, section 6 provides the conclusion.

Table 1 provide the list of notations which are used in this work.

\section{System model}

We assume a single cell Large Scale MU-MIMO system, where a base station equipped with $\mathrm{M}_{\mathrm{t}}$ transmitting antennas and $\mathrm{K}$ user equipments each having $\mathrm{N}_{\mathrm{r}}$ receive antennas as depicted in Fig. 1.

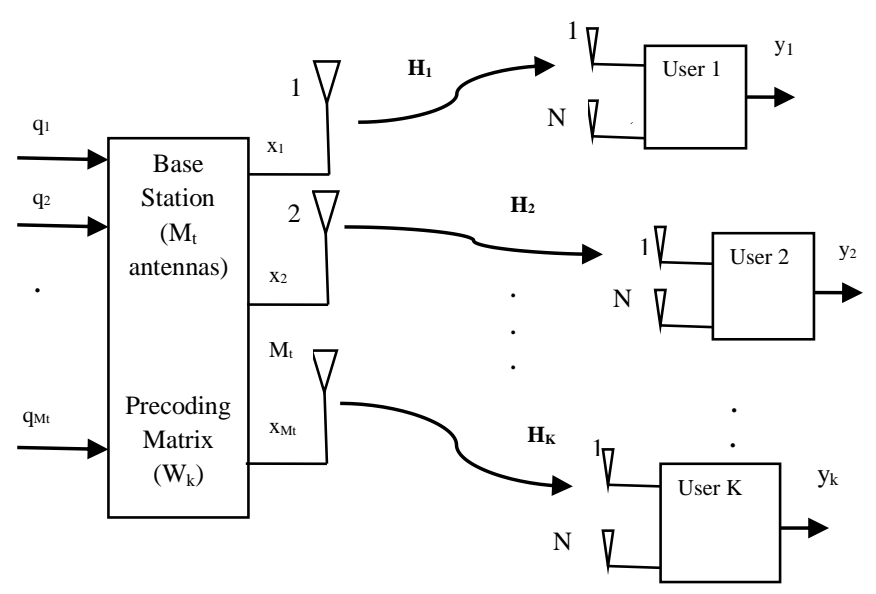

Figure. 1 Large scale MU-MIMO system 
The Rayleigh fading channel is modeled between $\mathrm{K}$ user terminals and base station (BS) antennas. The channel matrix is represented by $\mathrm{H} \epsilon$ $\mathrm{C}^{\mathrm{K} \times \mathrm{M}}$. We assume that perfect CSI is present at the base station for each user equipment. Multiple data streams $\mathrm{q}_{\mathrm{k}}$ are precoded for particular user $\mathrm{k}$ by precoding vector $\mathrm{W}_{\mathrm{k}}$. After precoding operation at the base station, these data streams for $\mathrm{K}$ user are combined and radiated from $\mathrm{M}_{\mathrm{t}}$ base station antennas.

The flat fading MIMO channel matrix among the base station antennas and user $\mathrm{k}$ can be written as

$$
H_{k}=\left[\begin{array}{ccccc}
h_{11} & h_{12} & \cdot & \cdot & h_{1 M_{t}} \\
h_{21} & h_{22} & \cdot & \cdot & h_{2 M_{t}} \\
\cdot & \cdot & \cdot & \cdot & \cdot \\
\cdot & \cdot & \cdot & \cdot & \cdot \\
h_{K 1} & h_{K 2} & \cdot & \cdot & h_{K M_{t}}
\end{array}\right]_{K X M_{t}}
$$

Here $\mathrm{H}_{\mathrm{k}} \in \mathrm{C}^{\mathrm{Nr} \times \mathrm{Mt}}$ represents MIMO channel matrix for a $\mathrm{k}^{\text {th }}$ user with distribution $C N \sim(0,1)$ which is considered to have full knowledge at the base station.

The signal received at $\mathrm{k}^{\text {th }}$ user is represented by

$$
y_{k}=H_{k} W_{k} q_{k}+H_{k} \sum_{i=1, i^{l} k}^{K} W_{i} q_{i}+n_{k}
$$

Here $\mathrm{q}_{\mathrm{k}}$ is transmitted signal for $\mathrm{k}^{\text {th }}$ user and $\mathrm{W}_{\mathrm{k}}$ is precoding matrix for user $\mathrm{k} . \mathrm{N}_{\mathrm{r}}$ data streams are transmitted by every user denoted by $\mathrm{q}_{\mathrm{k}} \in \mathrm{C}^{\mathrm{Nr} \times 1}$ and $\mathrm{n}_{\mathrm{k}} \in \mathrm{C}^{\mathrm{Nr} \times 1}$ represent the Gaussian noise for a $\mathrm{k}^{\text {th }}$ user with i.i.d. $C N \sim(0,1)$.

$$
\begin{aligned}
& W=\left[\begin{array}{lr}
W_{1} & W_{2} \ldots \ldots \ldots \ldots . W_{k}
\end{array}\right] \\
& q=\left[\begin{array}{ll}
q_{1} & q_{2} \ldots \ldots \ldots \ldots . . . q_{k}
\end{array}\right]^{T}
\end{aligned}
$$

Here $W \epsilon C^{M t x K N r}$ is the precoding matrix and $q \epsilon C^{K N r x l}$ is the total transmitted signal for $\mathrm{K}$ users. For simplification, equal power allocation is assumed and the power of individual stream is normalized to unity, then

$$
E\left[q q^{H}\right]=I_{N r}
$$

The channel matrix for all the users can be written as

$$
H_{s}=\left[H_{1}^{T} \ldots \ldots \ldots \ldots H_{k}^{T} \ldots \ldots \ldots \ldots H_{K}^{T}\right]^{T}
$$

Channel matrix excluding $\mathrm{k}^{\text {th }}$ user channel is as follows

$$
\tilde{H}_{k}=\left[H_{1}^{T} \ldots \ldots \ldots H_{k-1}^{T} \quad H_{k+1}^{T} \ldots \ldots \ldots . . H_{K}^{T}\right]^{T}
$$

Here $\quad \tilde{H}_{k} \in \square^{\stackrel{D}{r}_{r} \times M_{t}}$ and $\tilde{N}_{r}=N_{R}-N_{r}$

We represent $\mathrm{N}_{\mathrm{R}}=\mathrm{K}^{*} \mathrm{~N}_{\mathrm{r}}$.

\section{Proposed precoding algorithm}

In this section, we present a design of improved precoding algorithm for a LS-MU-MIMO system which employs $\mathrm{QR}$ decomposition and $\mathrm{ZF}$ based channel inversion (ZF-CI). The proposed algorithm is implemented in two stages. In the first stage, precoding matrix $\mathrm{W}^{\mathrm{I}}$ is derived to completely eliminate MUI. After elimination of MUI, MUMIMO channels are transformed into SU-MIMO channels. In the design of the proposed precoding algorithm, the orthonormal basis for zero space of matrix $\tilde{H}_{k}$ is determined by utilizing $\mathrm{QR}$ decomposition and pseudo-inverse. In the second stage of the proposed precoding algorithm, ZF-CI and $\mathrm{QR}$ decomposition operations are performed to obtain precoding matrix $\mathrm{W}^{\mathrm{II}}$ which parallelizes block channel into sub channels for users which further improve the precoding gain. The joint precoding matrix for the $\mathrm{k}^{\text {th }}$ user is given by

$$
W_{k, Q R-Z F-B D}=\left[W_{k}^{I} W_{k}^{I I}\right] \Lambda^{I / 2}
$$

Here $\wedge$ is the optimal power loading matrix.

\section{Stage I: Determination of first precoding matrix $\mathbf{W}^{\mathrm{I}}$}

The first precoding matrix is used to eliminate the MUI among users. For user k, apply ZF-CI on the matrix $\tilde{H}_{k}$ as

$$
\tilde{H}_{k, Z F}^{+}=\tilde{H}_{k}^{H}\left(\tilde{H}_{k} \tilde{H}_{k}^{H}\right)^{-1}
$$

Decompose $\tilde{H}_{k, Z F}^{\dagger}$ using QR decomposition operation, we get

$$
\tilde{H}_{k, Z F}^{\dagger}=Q_{k} R_{k}
$$

Where $R_{k}$ is the upper triangular matrix and invertible. $\mathrm{B}_{\mathrm{k}}$ is the rank of matrix $\tilde{H}_{k}$. Since $\mathrm{R}_{\mathrm{k}}$ is an invertible matrix, therefore, the expression 
$\tilde{H}_{k} Q_{k}=0$ holds true. Then $\left.Q_{k} \in C^{M}{ }_{t}{ }^{x}{ }_{t}{ }_{t}{ }_{k}\right)$ is the orthonormal basis for zero space of $\tilde{H}_{k}$.

From MUI mitigation constraint in Ref. [25], it is observed that $\mathrm{Q}_{\mathrm{k}}$ eliminates MUI and convert multi user-MIMO channel into parallel single userMIMO channels. Thus, the precoding matrix $W_{k}^{I}$ for the user $\mathrm{k}$ can be

$$
W_{k}^{I}=Q_{k}
$$

Similarly, the combined first precoding matrix $\mathrm{W}^{\mathrm{I}}$ is determined by implementing $\mathrm{QR}$ operations for all $\mathrm{K}$ users. Therefore, first precoding matrix for all the $\mathrm{K}$ users can be

$$
W^{I}=\left[W_{1}^{I} \ldots \ldots \ldots \ldots W_{k}^{I} \ldots \ldots \ldots \ldots . . W_{K}^{I}\right]
$$

\section{Stage II: Determination of second precoding matrix $\mathrm{W}^{\mathrm{II}}$}

After performing first the precoding process, Multi user-MIMO channel is converted into block channels for each user that are non-overlapping. Now the block channels are converted into SUMIMO parallel channels to optimize system performance. This can be achieved by the application of ZF-CI process on the equivalent channel matrix $H_{\text {Ileffk }}=\tilde{H}_{k} W_{k}^{I}$ as follows

$$
H_{I I e f f k, Z F}=\left(\tilde{H}_{k} W_{k}^{I}\right)^{H}\left[\left(\tilde{H}_{k} W_{k}^{I}\right)\left(\tilde{H}_{k} W_{k}^{I}\right)^{H}\right]^{-I}
$$

The equivalent matrix $F_{k}$ for user $k$ is

$$
F_{k}=W_{k}^{I} H_{I I e f f, Z F}
$$

With the increase in BS antennas and users for a LS-MU-MIMO system, the matrix dimensions become large and huge computations are required to determine its SVD. Therefore, it becomes beneficial to use $\mathrm{QR}$ decomposition operation on equivalent matrix $F_{k}$ instead of SVD operation in terms of computations required. Therefore, we decompose matrix $F_{k}$ using $Q R$ decomposition operation as

$$
F_{k}=Q_{I l k} R_{I I k}
$$

The second precoding matrix $W_{k}^{I I}$ for the user $\mathrm{k}$ can be

$$
W_{k}^{I I}=\left(Q_{I I k} \tilde{H}_{k}\right)
$$

Similarly, the combined precoding matrix $\mathrm{W}^{\mathrm{II}}$ is determined by implementing QR operation for all $\mathrm{K}$ users. Therefore, the overall second precoding matrix $\mathrm{W}^{\mathrm{II}}$ for all $\mathrm{K}$ users can be written as

$$
W^{I I}=\operatorname{diag}\left\{W_{1}^{I I}, \ldots \ldots \ldots \ldots . . W_{k}^{I I}, \ldots \ldots \ldots \ldots, W_{K}^{I I}\right\}
$$

Thus, the overall joint precoding matrix for QRZF-BD precoding algorithm is given by

$$
\begin{gathered}
W_{Q R-Z F-B D}=\left[W_{1}^{I} W_{1}^{I I}, \quad W_{2}^{I} W_{2}^{I I} \ldots \ldots \ldots . . W_{K}^{I} W_{K}^{I I}\right] \Lambda^{I / 2} \\
W_{Q R-Z F-B D}=\left[W^{I} W^{I I}\right] \Lambda^{1 / 2}
\end{gathered}
$$

The elements of the diagonal matrix $\wedge$ are used to scale the transmitted power into every column of

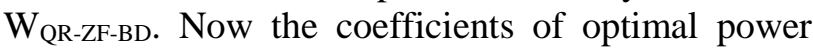
loading in $\wedge$ are found by application of water filling algorithm on the diagonal components of matrix $\boldsymbol{\Sigma}$ which contain singular values under power constraint P.

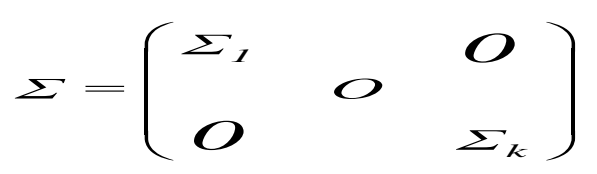

Where $\boldsymbol{\Sigma}_{\mathrm{k}}$ is the singular value matrix representing the scaling of right singular values.

The proposed QR-ZF-BD precoding is summarized in Algorithm 1.

\author{
Algorithm 1: Proposed QR-ZF-BD precoding \\ algorithm
}

1. Inputs:

Channel matrix H, SNR, K= number of users

2. Initialize:

3. Outputs:

Empty matrices $\boldsymbol{\Sigma}$ and $\mathbf{W}_{\text {ZF-QR-BD }}$

$\mathrm{W}_{\text {ZFQRBD }}$, Output signal y

4. for $\mathrm{k}=1: \mathrm{K}$ do

5. Apply ZF channel inversion

$$
\tilde{H}_{k, Z F}^{+}=\tilde{H}_{k}^{H}\left(\tilde{H}_{k} \tilde{H}_{k}^{H}\right)^{-1}
$$

6. Compute $\mathrm{QR}$ decomposition

$$
\tilde{H}_{k, Z F}^{\dagger}=Q_{k} R_{k}
$$

7. Compute first precoding matrix

$$
W_{k}^{I}=Q_{k}
$$


8. Compute equivalent matrix

$$
H_{\text {IIeffk }}=\tilde{H}_{k} W_{k}^{I}
$$

9. Apply ZF-CI operation

$$
H_{\text {IIeffk }, Z F}=\left(\tilde{H}_{k} W_{k}^{I}\right)^{H}\left[\left(\tilde{H}_{k} W_{k}^{I}\right)\left(\tilde{H}_{k} W_{k}^{I}\right)^{H}\right]^{-I}
$$

10. $F_{k}=W_{k}^{I} H_{\text {IIeff }, Z F}$

11. Compute $\mathrm{QR}$ decomposition

$$
F_{k}=Q_{I I k} R_{I I k}
$$

12. Compute second precoding matrix

$$
W_{k}^{I I}=\left(Q_{I I k} \tilde{H}_{k}\right)
$$

13.

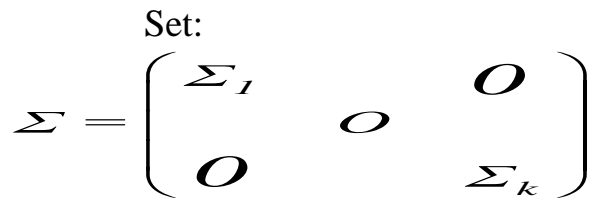

14. Compute power loading matrix $\Lambda=\operatorname{waterfill}(\Sigma)$

15. end for

16. Compute first overall precoding matrix

$$
W^{I}=\left[W_{1}^{I} \ldots \ldots \ldots \ldots W_{k}^{I} \ldots \ldots \ldots \ldots . W_{K}^{I}\right]
$$

17. Compute second overall precoding matrix

$$
W^{I I}=\operatorname{diag}\left\{W_{1}^{I I}, \ldots \ldots \ldots \ldots . . W_{k}^{I I}, \ldots \ldots \ldots \ldots, W_{K}^{I I}\right\}
$$

18. Joint precoding matrix for $\mathrm{K}$ users

$$
W_{Q R-Z F-B D}=\left[W^{I} W^{I I}\right] \Lambda^{1 / 2}
$$

\section{Performance analysis}

In this section, we provide the performance analysis of spectral efficiency (also called sum rate) for downlink single cell LS-MU-MIMO communication system with conventional ZF, MRT, MMSE, QR-BD, BD, and proposed QR-ZF-BD precoding algorithm. Generally, system capacity is considered as a popular method to measure the performance of MU-MIMO system since the increase in demand for high data rate services needs to be addressed.

The channel capacity over AWGN channel by Shannon can be written as

$$
C=\log _{2}(1+S N R) \mathrm{bit} / \mathrm{s} / \mathrm{Hz}
$$

In a multiuser MIMO interference is the addition of AWGN noise and interuser interference. Then the achievable rate for user $\mathrm{k}$ is

$$
c_{k}=\log _{2}\left(1+\operatorname{SINR} R_{k}\right) \mathrm{bit} / \mathrm{s} / \mathrm{Hz}
$$

Therefore, the spectral efficiency for a LS-MUMIMO communication system can be

$$
\begin{gathered}
C=\sum_{k=1}^{K} c_{k} \\
C=\sum_{k=1}^{K} \log _{2}\left(1+\operatorname{SINR}_{k}\right) \mathrm{bit} / \mathrm{s} / \mathrm{Hz}
\end{gathered}
$$

Eq. (22) is most commonly used for MU-MIMO communication system with Rayleigh fading channel [25, 37].

The spectral efficiency for QR-BD, BD, and proposed precoding algorithm is determined by [38]

$$
C=\log \left(\operatorname{det}\left(I+\sigma^{-2} H W W^{H} H^{H}\right)\right) \mathrm{bit} / \mathrm{s} / \mathrm{Hz}
$$

\section{Numerical results}

In this portion, we provide the performance of MRT, ZF, MMSE, QR-BD, BD, and proposed QRZF-BD precoding algorithms for LS-MU-MIMO downlink system by using Monte-Carlo simulations. In all the simulations, uncorrelated flat Raleigh fading and complex Gaussian noise with unit variance and zero mean is considered. We consider an equal number of antennas at every user terminal.

Fig. 2 depicts the spectral efficiency against the base station antennas $\mathrm{M}_{\mathrm{t}}$ for MRT, ZF, MMSE, BD, QR-BD, QR-ZF-BD precoding algorithms. In the simulation work, we consider the number of users $\mathrm{K}=15$ and each user having two antennas. The SNR considered in the simulation is $5 \mathrm{~dB}$. The base station antennas are varying from 10 to 100 in Fig. 2 and observe the effect on spectral efficiency for all precoding algorithms. It is observed from Fig. 2 that spectral efficiency increases with the increase in the number of base station antennas, which shows that LS-MU-MIMO system benefits dramatically the spectral efficiency. We simulate conventional

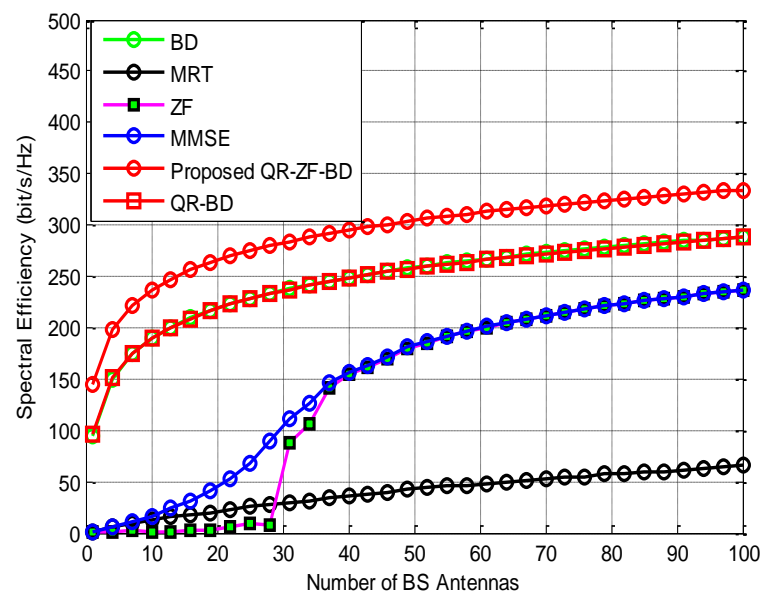

Figure. 2 Spectral efficiency vs number of antennas $\mathrm{M}_{\mathrm{t}}$ at BS for conventional and proposed precoder with $\mathrm{K}=15$, $\mathrm{Nr}=2$ 


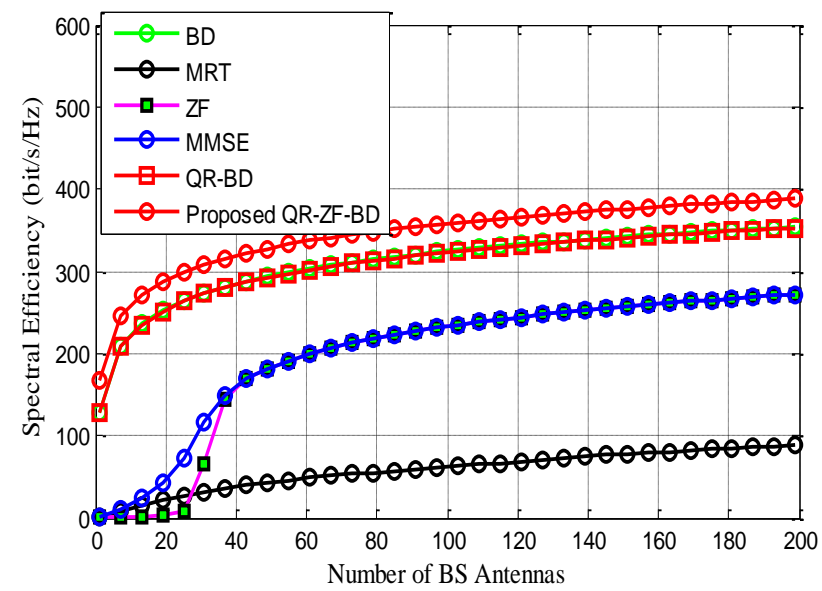

Figure. 3 Spectral efficiency vs number of antennas $\left(\mathrm{M}_{\mathrm{t}}\right)$ at BS for conventional and proposed precoder with $\mathrm{K}=15$, $\mathrm{Nr}=2$

linear precoding algorithms generally used in LSMU- MIMO system [11, 21, 26, 33] and compare with our proposed QR-ZF-BD precoding algorithm.

As observed from Fig. 2 that BD and QR-BD precoding algorithms provide the same performance but much improved as compared to MRT, MMSE, $\mathrm{ZF}$ precoding schemes. Fig. 2 depicts that the proposed QR-ZF-BD precoder outperformed the conventional $\mathrm{BD}, \mathrm{QR}-\mathrm{BD}, \mathrm{MRT}, \mathrm{ZF}$, and MMSE precoding algorithms. The improvement in spectral efficiency is due to ZF-CI operation on the equivalent matrix and $\mathrm{QR}$ decomposition operation in the second stage of the proposed algorithm.

Fig. 2 shows that MRT precoding provides poor performance as compared to other precoding algorithms but easier to implement. As observed from the results in Fig. 2 that the proposed algorithm performs excellent with low computational complexity, hence it becomes easier to implement proposed QR-ZF-BD precoder practically for a LSMU-MIMO communication system.

Fig. 3 shows the performance with 200 BS antennas. Further, Fig. 3 depicts that the performance of all precoding algorithms increases in terms of spectral efficiency with the increase in the number of antennas at BS as compared to Fig. 2. In addition to that, we also note that there is a substantial improvement in the spectral efficiency of the proposed precoding algorithm as compared to other precoding algorithms $[21,33]$.

We plot spectral efficiency graphs with different SNR values. Figs. 4, 5, 6 and 7 depicts the spectral efficiency versus SNR for MMSE, MRT, ZF, QR$\mathrm{BD}, \mathrm{BD}$, and proposed $\mathrm{QR}-\mathrm{ZF}-\mathrm{BD}$ precoding algorithms.

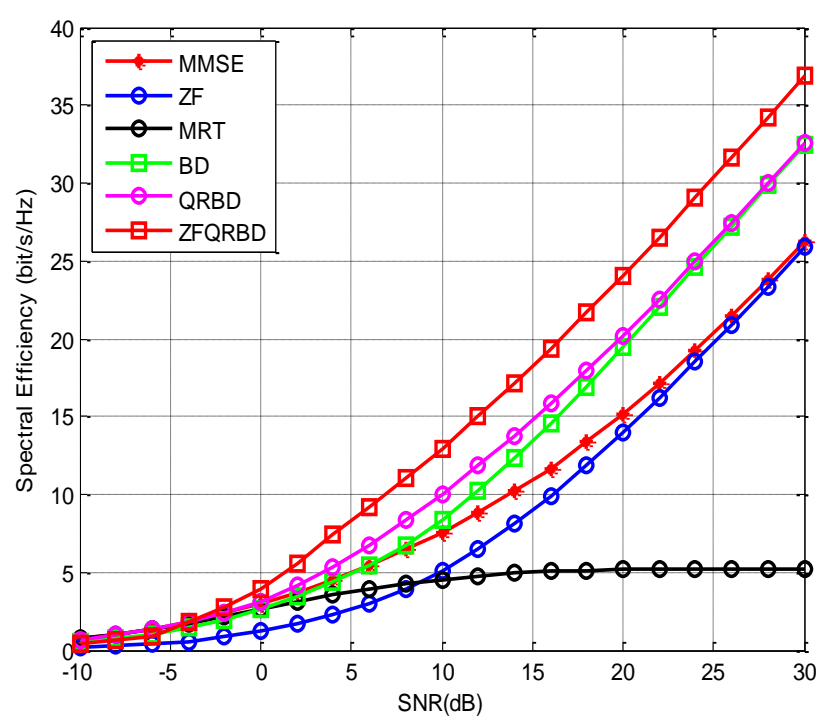

Figure. 4 Spectral efficiency comparison as a function of SNR Values for proposed and conventional precoder with

$$
\mathrm{M}_{\mathrm{t}}=4, \mathrm{~K}=2 \text {, and } \mathrm{N}_{\mathrm{r}}=2
$$

Fig. 4 shows the results for $\mathrm{M}_{\mathrm{t}}=4$ base station antennas, we observe that MRT precoding is near optimal at low SNRs and ZF precoding scheme is optimal at high values of SNRs. MMSE precoding is a more versatile scheme that collaborates the respective properties of MRT and ZF precoding schemes with better performance at intermediate SNR values.

The performance of the BD precoding approaches to that of $\mathrm{QR}-\mathrm{BD}$ precoding scheme with the increase in SNR values and proposed precoding algorithm performs well at all SNR range as depicted from Fig. 4. The aim of the simulation results depicted in Fig. 4 is to study the behavior of MRT, ZF, MMSE precoding schemes clearly at low SNR values and with less number of BS antennas. MRT precoding scheme is performing well at low SNRs.

Fig. 5 shows the performance with $M_{t}=12$ base station antennas, in this case, base station antennas are more than the number of users and MMSE precoding is nearly optimal in the entire SNR regime. As antennas at BS increases, ZF and MMSE precoding show the same performance. Similar behavior is observed for QR-BD and $\mathrm{BD}$ precoding algorithms. This is the important observation for the system with a higher number of antennas at base station i.e in LS-MU-MIMO system. Proposed precoding design in this scenario also shows better performance in the entire SNR range. 


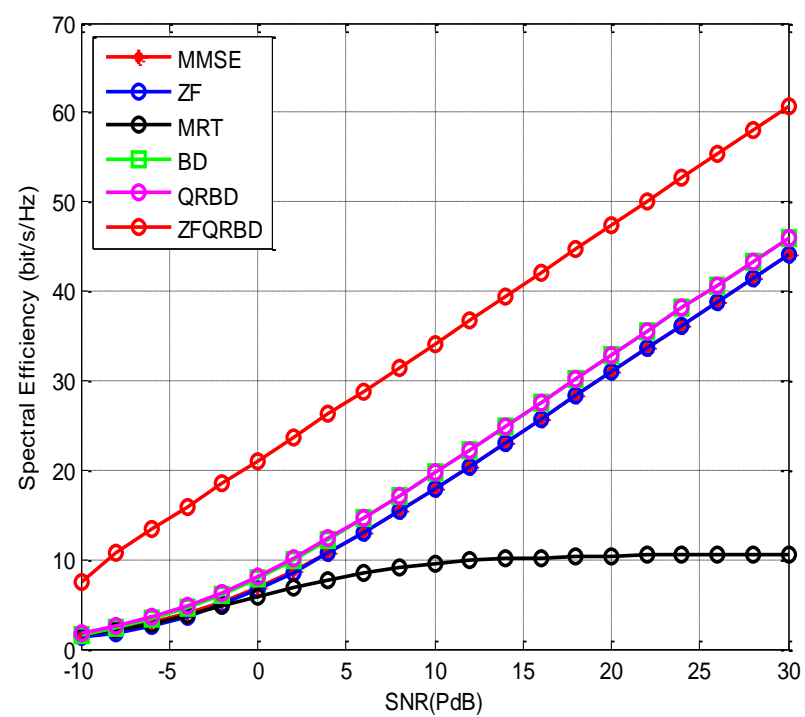

Figure. 5 Spectral efficiency comparison as a function of SNR Values for proposed and conventional precoder with $\mathrm{M}_{\mathrm{t}}=12, \mathrm{~K}=2$, and $\mathrm{N}_{\mathrm{r}}=2$

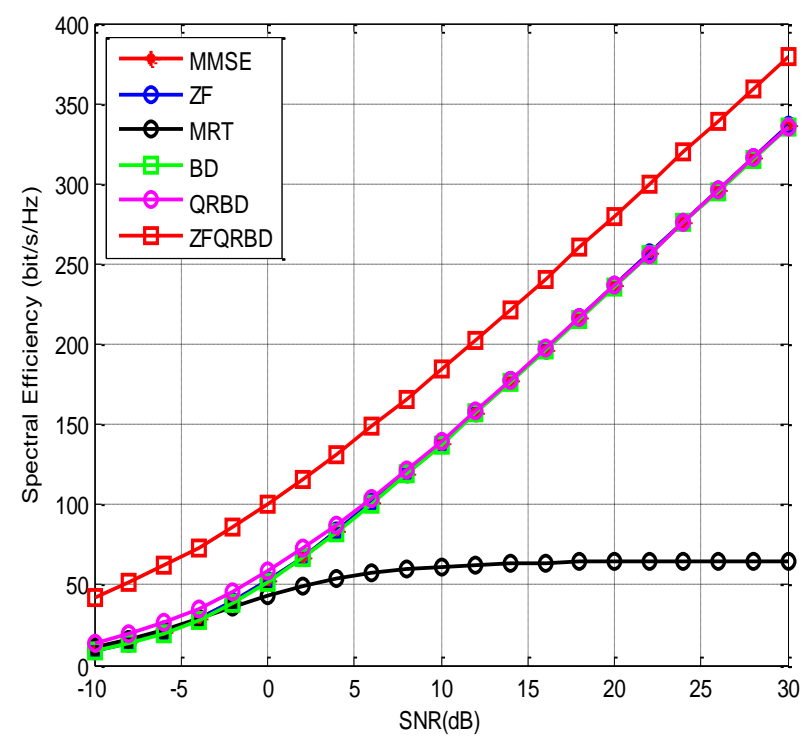

Figure. 6 Spectral efficiency comparison as a function of SNR Values for proposed and conventional precoder with $\mathrm{M}_{\mathrm{t}}=100, \mathrm{~K}=15$, and $\mathrm{N}_{\mathrm{r}}=2$

Fig. 6 depicts the spectral efficiency versus different SNR values for MRT, MMSE, ZF, BD, QR-BD and proposed QR-ZF-BD precoding algorithm. We consider $\mathrm{K}=15$ users having two antennas on each, antennas at base station $\mathrm{M}_{\mathrm{t}}=100$ and SNR is varying from -10 to $30 \mathrm{~dB}$. It is observed from the Fig. 6 that at low SNR values the spectral efficiency of MRT precoding is higher than ZF precoding. This fact is also clearly observed from Fig. 4. Therefore, MRT precoding scheme is the better option when the transmitter emits low power.

As shown from Figs. 4, 5, 6 and 7 that the

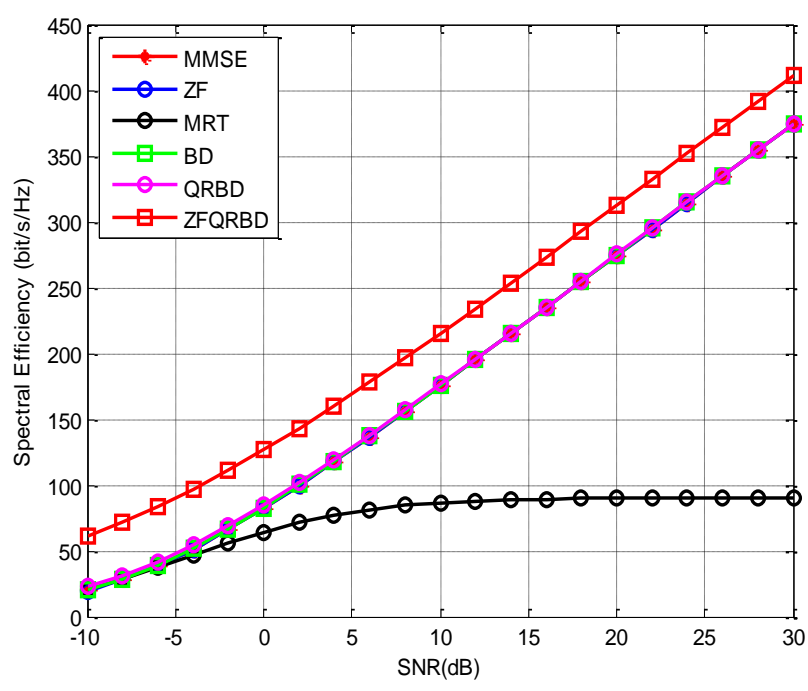

Figure. 7 Spectral efficiency comparison as a function of SNR Values for proposed and conventional precoder with $\mathrm{M}_{\mathrm{t}}=200, \mathrm{~K}=15$, and $\mathrm{N}_{\mathrm{r}}=2$

performance of MRT precoding saturated with increasing SNR values due to poor interference elimination capability as compared to ZF, MMSE, QR-BD, BD, and QR-ZF-BD precoding algorithms.

At high SNR values, the spectral efficiency with ZF, MRT, BD, QR-BD, and QR-ZF-BD outperforms MRT precoding as depicted from Fig. 6. It is observed from Fig. 6 that our proposed QRZF-BD precoding algorithm achieves higher spectral efficiency in the entire SNR region as compared to the conventional precoders. It is noted that at higher SNR region the ZF, MMSE approaches to the performance of $\mathrm{QR}-\mathrm{BD}$ and $\mathrm{BD}$ precoding techniques. Also, the $\mathrm{BD}$ and $\mathrm{QR}-\mathrm{BD}$ precoding show a similar performance but with less complexity of QR-BD precoding as compared to BD precoding. The improved performance of the proposed precoding algorithm is due to a further reduction in interference by $\mathrm{ZF}-\mathrm{CI}$ and $\mathrm{QR}$ decomposition operation.

Fig. 7 shows the performance with $\mathrm{M}_{\mathrm{t}}=200$ antennas, $K=15$ users, $N_{r}=2$. We observe that the addition of more number of antennas at the BS provides additional spectral efficiency for all precoding algorithms. In whole span of SNRs, proposed QR-ZF-BD precoding algorithm outperforms as compared to all other precoding algorithms [37].

The proposed QR-ZF-BD precoding algorithm can fulfill the demand for higher data rates in the next-generation communication system. The IMTAdvanced which specifies the spectral efficiency in the range of $2-3 \mathrm{bit} / \mathrm{s} / \mathrm{Hz} / \mathrm{cell}$ for $4 \mathrm{G}$ communication system [39]. In comparison, LS-MU-MIMO system considered in Fig. 3 attains spectral efficiencies of 
values $88,271,272,353,388 \mathrm{bit} / \mathrm{s} / \mathrm{Hz}$ with $\mathrm{MRT}$, ZF, MMSE, BD, and proposed precoding algorithm respectively using 200 base station antennas and 15 two antenna users. This attained value of spectral efficiency with proposed design is $129 \mathrm{x}$ to $194 \mathrm{x}$ enhancement as compared to IMT-Advanced. From these numerical results, we say that the proposed QR-ZF-BD precoding algorithm can be practically implemented to provide improved spectral efficiency for downlink LS-MU-MIMO system.

\section{Conclusion}

We proposed QR-ZF-BD precoding algorithm design for the downlink of a LS-MU-MIMO system where users having multi-antennas. In this work, the spectral efficiency for a single-cell downlink LSMU-MIMO communication system with Rayleigh fading channel has been investigated, which considers different types of precoding techniques. The proposed QR-ZF-BD precoding algorithm utilizes QR operation to determine zero space vectors to eliminate MUI, instead of SVD operation which reduces the implementation complexity of the proposed precoding algorithm as compared to the conventional Block Diagonalization precoding algorithm. The precoding gain is further enhanced with ZF-CI and QR operations which reduce the interference between the users. The spectral efficiency of the proposed ZF-QR-BD precoding algorithm has been analyzed and compared with conventional MRT, ZF, MMSE, QR-BD, BD, precoding algorithms. Numerical results indicate that the proposed precoding algorithm attains substantial improvement in performance gain as compared to LTE system [39] and other precoding algorithms with lesser computational complexity. We conclude that our proposed QR-ZF-BD precoding algorithm is considerable for future generation LS-MU-MIMO communication system. In future work, we analyze the proposed precoding algorithm for multicell MU-MIMO system to deal with the pilot contamination problem.

\section{References}

[1] E. G. Larsson, O. Edfors, F. Tufvesson, and T. L. Marzetta, "Massive MIMO for next generation wireless systems", IEEE Communications Magazine, Vol. 52, No. 2, pp. 186-195, 2014.

[2] F. Boccardi, R. W. Heath, A. Lozano, T. L. Marzetta, and P. Popovski, "Five disruptive technology directions for 5G", IEEE Communications Magazine, Vol. 52, No. 2, pp. 74-80, 2014.
[3] Ericson Mobility Report on the Pulse of the Network Society, Revision A, 2016, pp. 1-32.

[4] Cisco, Cisco visual networking index: Global mobile data traffic forecast update, 2016-2021, 2017.

[5] T. L. Marzetta, "Massive MIMO: An Introduction", Bell Labs Technical Journal, Vol. 20, pp. 11-22, 2015.

[6] D. Gesbert, M. Kountouris, R. W. Heath, C. Chae, and T. Salzer, "Shifting the MIMO Paradigm”, IEEE Signal Processing Magazine, Vol. 24, No. 5, pp. 36-46, 2007.

[7] F. Rusek, D. Person, B. K. Lau, E. G. Larsson, T.L. Marzetta, O. Edfors, and F. Tufvesson, "Scaling Up MIMO: Opportunities and Challenges with Very Large Arrays", IEEE Signal Processing Magazine, Vol. 30, No. 1, pp. 40-60, 2013.

[8] S. Parkvall, E. Dahlman, A. Furuskar, and M. Frenne, "NR: The New 5G Radio Access Technology", IEEE Communications Standards Magazine, Vol. 1, No. 4, pp. 24-30, 2017.

[9] E. Björnson, E. G. Larsson, and T. L. Marzetta, "Massive MIMO: ten myths and one critical question", IEEE Communications Magazine, Vol. 54, No. 2, pp. 114-123, 2016.

[10] T. L. Marzetta, "Noncooperative Cellular Wireless with Unlimited Numbers of Base Station Antennas", IEEE Transactions on Wireless Communications, Vol. 9, No. 11, pp. 3590-3600, 2010.

[11] H. Q. Ngo, E. G. Larsson, and T. L. Marzetta, "Energy and Spectral Efficiency of Very Large Multiuser MIMO Systems", IEEE Transactions on Communications, Vol. 61, No. 4, pp. 14361449, 2013.

[12] X. Gao, F. Tufvesson, and O. Edfors, "Massive MIMO channels - Measurements and models", In: Proc. of 2013 Asilomar Conference on Signals, Systems and Computers, pp. 280-284, 2013.

[13] O. N. Alrabadi, E. Tsakalaki, H. Huang, and G. F. Pedersen, "Beamforming via Large and Dense Antenna Arrays Above a Clutter", IEEE Journal on Selected Areas in Communications, Vol. 31, No. 2, pp. 314-325, 2013.

[14] L. Zhao, K. Zheng, H. Long, and H. Zhao, "Performance analysis for downlink massive MIMO system with ZF precoding", Transaction on Emerging Telecommunication Technologies, Vol. 25, pp. 1219-1230, 2014.

[15] W. Tan, X. Feng, G. Liu, W. Tan, M. Zhou, and C. Li, "Spectral Efficiency of Massive MIMO Systems With Multiple Sub-Arrays 
Antenna", IEEE Access, Vol. 6, pp. 3121331223, 2018.

[16] J. Jose, A. Ashikhmin, T. L. Marzetta, and S. Vishwanath, "Pilot Contamination and Precoding in Multi-Cell TDD Systems", IEEE Transactions on Wireless Communications, Vol. 10, No. 8, pp. 2640-2651, 2011.

[17] H. Yin, D. Gesbert, M. Filippou, and Y. Liu, "A Coordinated Approach to Channel Estimation in Large-Scale Multiple-Antenna Systems", IEEE Journal on Selected Areas in Communications, Vol. 31, No. 2, pp. 264-273, 2013.

[18] M. Zhang, W. Tan, J. Gao, and S. Jin, "Spectral efficiency and power allocation for mixed-ADC massive MIMO system", China Communications, Vol. 15, No. 3, pp. 112-127, 2018.

[19] M. Zhao, D. Deng, W. Zhou, and L. Fan, "Non-renewable energy efficiency optimization in energy harvesting relay-assisted system", Physical Communication, Vol. 29, pp. 183-190, 2018.

[20] W. Tan, S. Jin, C. K. Wen, and J. Tao, "Spectral efficiency of multi-user millimetre wave systems under single path with uniform rectangular arrays", EURASIP Journal on Wireless Communications and Networking, Vol. 181, pp. 458-472, 2017.

[21] Y. Lim, C. Chae, and G. Caire, "Performance Analysis of Massive MIMO for Cell-Boundary Users", IEEE Transactions on Wireless Communications, Vol. 14, No. 12, pp. 68276842, 2015.

[22] E. Björnson, L. Sanguinetti, J. Hoydis, and M. Debbah, "Optimal Design of Energy-Efficient Multi-User MIMO Systems: Is Massive MIMO the Answer?", IEEE Transactions on Wireless Communications, Vol. 14, No. 6, pp. 30593075, 2015.

[23] C. Li, K. Song, Y. Li, and L. Yang, "Energy efficient design for multiuser downlink energy and uplink information transfer in 5G", Science China Information Sciences, Vol. 59, No. 2, pp. 30-48, 2016.

[24] Q. Zhang, S. Jin, K. Wong, H. Zhu, and M. Matthaiou, "Power Scaling of Uplink Massive MIMO Systems With Arbitrary-Rank Channel Means", IEEE Journal of Selected Topics in Signal Processing, Vol. 8, No. 5, pp. 966-981, 2014.

[25] Q. H. Spencer, A. L. Swindlehurst, and M. Haardt, "Zero-forcing methods for downlink spatial multiplexing in multiuser MIMO channels", IEEE Transactions on Signal Processing, Vol. 52, No. 2, pp. 461-471, 2004.

[26] T. X. Tran, and K. C. Teh, "Performance analysis of massive multiuser multiple-input multiple-output systems with block diagonalisation", IET Communications, Vol. 10, No. 7, pp. 832-838, 2016.

[27] Z. Li, Y. Liu, Y. Zhang, P. Wang, C. Tian, and $X$. Sha, "Hybrid coordinated strategy of downlink coordinated multi-point transmission", Journal of Systems Engineering and Electronics, Vol. 26, No. 5, pp. 916-923, 2015.

[28] D. H. N. Nguyen, H. Nguyen-Le, and T. LeNgoc, "Block-Diagonalization Precoding in a Multiuser Multicell MIMO System: Competition and Coordination", IEEE Transactions on Wireless Communications, Vol. 13, No. 2, pp. 968-981, 2014.

[29] H. Sung, S. Lee, and I. Lee, "Generalized Channel Inversion Methods for Multiuser MIMO Systems", IEEE Transactions on Communications, Vol. 57, No. 11, pp. 34893499, 2009.

[30] J. Wu, S. Fang, L. Li, and Y. Yang, "QR decomposition and Gram Schmidt Orthogonalization based low-complexity multiuser MIMO precoding”, In: Proc. of the 10th International Conference on Wireless Communications, Networking and Mobile Computing, pp. 61-66, 2014.

[31] L. Tran, M. Bengtsson, and B. Ottersten, "Iterative Precoder Design and User Scheduling for Block-Diagonalized Systems", IEEE Transactions on Signal Processing, Vol. 60, No. 7, pp. 3726-3739, 2012.

[32] H. Wang, L. Li, L. Song, and X. Gao, "A Linear Precoding Scheme for Downlink Multiuser MIMO Precoding Systems", IEEE Communications Letters, Vol. 15, No. 6, pp. 653-655, 2011.

[33] Y. Zhang, J. Gao, and Y. Liu, "MRT precoding in downlink multiuser MIMO systems", EURASIP Journal on Wireless Communications and Networking, No. 1, pp. 241, 2016.

[34] K. Zu, R. C. de Lamare, and M. Haardt, "Generalized Design of Low-Complexity Block Diagonalization Type Precoding Algorithms for Multiuser MIMO Systems", IEEE Transactions on Communications, Vol. 61, No. 10, pp. 42324242, 2013.

[35] M. H. A. Khan, K. M. Cho, M. H. Lee, and M. H. Mustary, "Multi-Cell block diagonalization precoding for multiuser MIMO broadcast channel", In: Proc. of 2014 IEEE International 
Symposium on Broadband Multimedia Systems and Broadcasting, pp. 1-6, 2014.

[36] M. Maleki, K. Mohamed-Pour, and M. Soltanalian, "Receive Spatial Modulation in Correlated Massive MIMO With Partial CSI", IEEE Transactions on Signal Processing, Vol. 67, No. 5, pp. 1237-1250, 2019.

[37] E. Björnson, M. Bengtsson, and B. Ottersten, "Optimal Multiuser Transmit Beamforming: A Difficult Problem with a Simple Solution Structure [Lecture Notes]", IEEE Signal Processing Magazine, Vol. 31, No. 4, pp. 142148, 2014.

[38] S. Vishwanath, N. Jindal, and A. Goldsmith, "On the capacity of multiple input multiple output broadcast channels", In: Proc. of 2002 IEEE International Conference on Communications. Conference Proceedings, pp. 1444-1450, 2002.

[39] I. F. Akyildiz, D. M. Gutierrez-Estevez, and E. C. Reyes, "The evolution to $4 \mathrm{G}$ cellular systems: LTE-Advanced", Physical Communication, Vol. 3, No. 4, pp. 217-244, 2010. 